\title{
PROJECTIVE MODULES AS FIBER BUNDLES ${ }^{1}$
}

\author{
E. GRAHAM EVANS, JR.
}

ABstract. Projective modules over real affine rings, $R$, with compact real points, $X$, can be considered as fiber bundles over $X$ by tensoring with the real valued continuous functions on $X$. This induces a map $f: K_{0}(R) \rightarrow K^{0}(X)$. In this article the kernel of $f$ is determined and an example with nonzero kernel is given.

Introduction. In [3] Swan constructs a correspondence between projective modules and fiber bundles and uses it to construct several examples of projective modules over affine rings whose properties mimic the properties of the fiber bundles. He also suggests that nontrivial projectives could become free in the process $[3$, p. 273]. More recently Fossum [1] has shown that for spheres every bundle can be obtained in this way. He refers to the example at the end of this note. This investigation began to try to find some criteria on a real affine ring $R$ with compact real points, $X$, so that the map $K_{0}(R) \rightarrow K^{0}(X)$ would be an isomorphism. $R$ regular was considered a test case. The example given shows failure even for $R$ Dedekind. All rings are commutative with unit. See [2] for more detail about algebraic $K$ theory.

The author thanks R. G. Swan for suggesting the problem and for his assistance in solving it.

THEOREM 1. Let $R C C$ be rings where $C$ is a topological ring whose elements can be approximated by elements of $R$ and whose units are an open set. Then if $S=\{r \in R \mid r$ is a unit in $C\}$. The map $K_{0}\left(R_{S}\right) \rightarrow K_{0}(C)$ is a monomorphism.

Proof. Let $[P]-[Q] \in \operatorname{ker}\left(K_{0}\left(R_{S}\right) \rightarrow K_{0}(C)\right)$. By adding a finitely generated complement of $Q$ to $P$ and $Q$ we can assume $Q$ is free. Then by adding on a finitely generated free module to $P$ and $Q$ we can assume $P$ is isomorphic to a free module over $C$. Hence it is enough to prove the following:

Received by the editors October 14, 1969.

AMS 1969 subject classifications. Primary 5730, 1390, 1340; Secondary 1315, 1401, $1320,1820$.

Key words and phrases. Projective modules, fiber bundles, topological ring, real affine rings, norms.

1 Preparation of this paper was supported in part by NSF Grant No. GP-9661, most of it was written while the author held a NASA traineeship at the University of Chicago. 
Theorem 2. Let $R, C$, and $S$ be as above. Let $P$ be a finitely generated projective $R_{S}$ module which is free over $C$. The $P$ is free.

Proof. Let $Q$ be a finitely generated complement to $P$. By adding on to $Q$ a free module of rank the rank of $P$ we can assume that $Q$ becomes free over $C$. Hence we have $Q \oplus P$ free where $Q$ is the image of an idempotent matrix $e_{Q}$ and $P$ is the image of the idempotent matrix $e_{P}=1-e_{Q}$. Over $C, Q$ becomes isomorphic to a free, $F$, and $P$ to a free, $F^{\prime} . F$ and $F^{\prime}$ are the images of idempotent matrices $e_{F}=\left(e_{F_{i j}}\right)$ and $e_{F^{\prime}}=1-e_{F}$ where $e_{F_{i j}}=0$ if $i \neq j, e_{F_{i i}}=1$ if $i=1,2, \cdots, n$ where $n$ is the rank of $F$ and $e_{F_{i i}}=0 i=n+1, \cdots, n+m$ where $m$ is the rank of $F^{\prime}$. Hence over $C$ we have a commutative diagram with $g$ restricted to $Q, g$ restricted to $P$ to $g$ all isomorphisms (with $F, F^{\prime}$, and $F \oplus F^{\prime}$ respectively):

$$
\begin{aligned}
& 0 \rightarrow P \rightarrow P \oplus Q \rightarrow Q \rightarrow 0 \\
& \downarrow g \quad \downarrow g \downarrow g \\
& 0 \rightarrow F^{\prime} \rightarrow F^{\prime} \oplus F \rightarrow F \rightarrow 0 .
\end{aligned}
$$

Then $g=e_{F} g e_{Q}+e_{F} g e_{P} . g$ is a matrix with entries in $C$. We approximate $g$ by $g_{1}, g_{2}, \cdots$ which have entries in $R$. We form $g_{i}^{\prime}=e_{F} g e_{Q}$ $+e_{F^{\prime}} g e_{P}$. The $g^{\prime}$ also have entries in $R$ and approximate $g . g_{i}^{\prime}$ is an isomorphism if $\operatorname{det}\left(g_{i}^{\prime}\right)$ is a unit. The units are open. Hence from some $n$ on $g_{i}^{\prime}$ is an isomorphism. But $g_{i}^{\prime}$ maps $P$ to $F^{\prime}$. Hence from $n$ on $g_{i}^{\prime}$ restricted to $P$ is an isomorphism of $P$ with $F^{\prime}$ which has entries in $R$. Hence $\operatorname{det}\left(g_{i}^{\prime}\right) \in S$ and $g$ is an isomorphism of $P$ with a free module over $R_{S}$. This completes the proof of Theorems 1 and 2.

REMARK. Theorems 1 and 2 are applicable if $R$ is real affine with compact real points, $X$, and $C$ is the real valued continuous functions on $X$ or if $R$ is the differentiable real (complex) valued function on a compact real (complex) manifold and $C$ is the continuous real (complex) valued functions.

Corollary 3. With $R, S$, and $C$ as above the kernel of $K_{0}(R) \rightarrow K_{0}(C)$ is generated by all $[P]-[F]$ where $F$ is free and $P$ is isomorphic to $F$ over $R_{S}$.

Given Theorem 1 the search for an example was easy. By picking $R$ Dedekind the stable difficulties are removed. Following Swan [3, p. 273], we picked an ideal, $I$, going to the möbius band whose square is not principal over $R$ but is principal over $R_{S}$.

ExAmple. Let $R=R[X, Y] /\left(Y^{2}-\left(1-X^{2}\left(X^{2}+1\right)\right)\right)$ where $R$ is the reals. It is easy to check that $R$ is a Dedekind domain whose real 
points are topologically a circle. The ideal $(1-Y, X)$ goes to the möbius band $(X, 1-Y)^{2}=\left(X^{2}, 1-Y\right)$. Thus, $\left(X^{2}, 1-Y\right)=(1-Y)$ over any ring in which $X^{2}+1$ is unit. Hence it is enough to show $\left(X^{2}, 1-Y\right)$ is not principal in $R$. First we need a few lemmas.

\section{LemMa 3. $X$ is irreducible in $R$.}

Proof. We use the norm function

$$
N(a+b Y)=a^{2}-b^{2}\left(1-X^{2}\left(X^{2}+1\right)\right) .
$$

Suppose that $X=\left(r_{0}+r_{1} Y\right)\left(s_{0}+s_{1} Y\right)$ with $r_{i}, s_{i} \in R[X]$. To eliminate the trivial factorization we can suppose $r_{1}$ (and hence $s_{1}$ ) is nonzero. Taking norms we have

$$
X^{2}=\left(r_{0}^{2}-r_{1}^{2}\left(1-X^{2}\left(X^{2}+1\right)\right)\right)\left(s_{0}^{2}-s_{1}^{2}\left(1-X^{2}\left(X^{2}+1\right)\right)\right) .
$$

It is enough to eliminate the possibility $r_{0}^{2}-r_{1}^{2}\left(1-X^{2}\left(X^{2}+1\right)\right)=c X$ with $c \in R-\{0\}$, for if $N\left(r_{0}+r_{1} Y\right)$ is a unit of $R[X]$ then $r_{0}+r_{1} Y$ is a unit of $R$. Suppose $r_{0}^{2}-r_{1}^{2}\left(1-X^{2}\left(X^{2}+1\right)\right)=c X$. Then factoring out by the positive real root of $1-X^{2}\left(X^{2}+1\right)$ shows $c$ is positive. But factoring out by the negative root of $1-X^{2}\left(X^{2}+1\right)$ shows $c$ is negative. Hence $X$ is irreducible.

Lemma 4. $X^{2}$ has only factorizations involving $X$ (up to units of $R$ ).

Proof. Say $X^{2}=\left(r_{0}+r_{1} Y\right)\left(s_{0}+s_{1} Y\right)$ with $r_{i}, s_{i} \in R[X]$. Then $r_{1}$ (and hence $s_{1}$ ) can be assumed nonzero as before. Taking norms we have

$$
X^{4}=\left(r_{0}^{2}-r_{1}^{2}\left(1-X^{2}\left(X^{2}+1\right)\right)\right)\left(s_{0}^{2}-s_{1}^{2}\left(1-X^{2}\left(X^{2}+1\right)\right)\right) .
$$

We already know that $r_{0}^{2}-r_{1}^{2}\left(1-X^{2}\left(X^{2}+1\right)\right)$ cannot be $c X$ with $c \in R-\{0\}$. Hence it is enough to examine the case $r_{0}^{2}-$ $r_{1}^{2}\left(1-X^{2}\left(X^{2}+1\right)\right)=c X^{2}$ with $c \in R-\{0\}$. Examining the highest nonzero term of both sides we see that (highest $\neq 0$ term of $\left.r_{0}\right)^{2}+$ (highest $\neq 0$ term of $\left.r_{1}\right)^{2}=0$. Hence both parts are 0 which is a contradiction.

Finally we show that $\left(1-Y, X^{2}\right)$ is not principal. If it were, then generator, $g$, would necessarily divide $X^{2}$. Since $\left(1-Y, X^{2}\right)$ is a proper ideal, $g$, is not a unit. Hence, up to units, $g$ is either $X$ or $X^{2}$. But $1-Y$ is not a multiple of $X$ since we can send $X$ to 0 and $1-Y$ to 2. This completes the example.

The following example suggested by Kaplansky shows that $K_{0}(R) \rightarrow K^{0}(X)$ can fail to be onto. Let $f(X)$ be a real quartic with distinct real roots and such that the coefficient of $X^{4}$ is negative. 
Then $R=R[X, Y] /\left(Y^{2}-f(X)\right)$ is a domain. The real points of $R$ form two disjoint circles. Hence there are bundles with different fiber dimensions on the different circles. These cannot come from $R$ since $R$ is a domain and the rank of projectives is constant.

\section{REFERENCES}

1. Robert M. Fossum, Vector bundles over spheres are algebraic, Invent. Math. 8 (1969), 222-225. MR 40 \#3537.

2. R. G. Swan, Algebraic $K$-theory, Lecture Notes in Math., no. 76, SpringerVerlag, Berlin, 1968. MR 39 \#6940.

3. - Vector bundles and projective modules, Trans. Amer. Math. Soc. 105 (1962), 264-277. MR 26 \#785.

University of Chicago, Chicago, Illinois 60637

University of California, Los Angeles, California 90024

Massachusetts Institute of Technology, Cambridge, Massachusetts 02139 